\title{
Results on soft continuous functions in the soft topological spaces equipped with soft Scott topology
}

\author{
Gözde Yaylalı*, Bekir Tanay \\ Department of Mathematics, Faculty of Science, Muğla Sıtkı Koçman University, Muğla, Turkey. \\ Communicated by E. Savas
}

\begin{abstract}
In this study, some properties of soft Scott topology are examined and some relations between soft Scott topology and way below soft set relations are shown. Also the notion of soft Scott continuous function on soft topological spaces, which is equipped with soft Scott topology, is defined by focusing on the structure of the continuity of soft function and some examples are illustrated. Besides these, least fixed point theorem is proved for soft Scott continuous functions. @ 2017 All rights reserved.
\end{abstract}

Keywords: Soft set, soft topology, soft continuous function, soft Scott topology, least fixed point theorem. 2010 MSC: 03E72, 54J99, 54A40, 54A99, 47H10.

\section{Introduction}

In the real world, most of problems in economics, engineerings and environmental areas can be solved approximately by using different mathematical theories. Besides these theories, Molodtsov [8] introduced a new approach, the soft set theory, which approximates the initial universe for the problems. In the soft set theory, one can use any parametrization with the help of words, sentences, real numbers, functions, mappings, and so on. Maji et al. [7] introduced many operators for soft set theory. Furthermore, some structures as cartesian product, soft set relation and orderings on soft sets was introduced by Babitha and Sunil $[2,3]$. Moreover, Tanay and Yaylalı improved orderings on soft sets by giving new definitions and proving new theorems on some soft structures such as directed complete soft sets and way below soft set relation [12, 17, 16, 13]. Çağman et al. [4] defined soft topology by modifying the definition of soft set. After this study Roy and Samanta [10] strengthened the definition of soft topological spaces. Tanay and Yaylalı [12] gave the definition of soft Scott topology by using orderings and soft topology on soft sets. Wardowski [14] approached soft set as classical mathematics by giving definition of soft element. By giving this definition, he strengthened the soft function and gave the continuity of soft functions.

According to all of these studies, in this study, firstly we define an ordering on soft elements, then we use soft continuity to define and examine soft Scott continuous functions. Also, we prove least fixed point theorem for soft Scott continuous functions. In order to refresh the fundamental concepts we refer to $[5,6]$.

\footnotetext{
${ }^{*}$ Corresponding author

Email addresses: gozdeyaylali@mu.edu.tr (Gözde Yaylalı), btanay@mu.edu.tr (Bekir Tanay)
} 


\section{Preliminaries and basic definitions}

Definition 2.1 ([8]). Let $U$ be an initial universe and $E$ be the set of parameters. Let $\mathcal{P}(U)$ be the set of all subsets of $U$ and $A$ be a subset of $E$. A pair $(F, A)$ is called a soft set over $U$ where $F: A \longrightarrow \mathcal{P}(U)$ is a set-valued function.

In some studies a soft set $(F, A)$ was shown as $(F, A)=\{(a, F(a)) \mid a \in A\}$, but in some studies $F(a)$ was written instead of $(a, F(a))$ just as a notation for make it shorter. In this paper, we will use $F(a)$ as a notation instead of $(a, F(a))$.

All soft sets over the initial universe $U$ is denoted by $S(U)$ throughout this paper.

Definition $2.2([7])$. A soft set $(F, A)$ over $U$ is said to be a null soft set denoted by $\Phi$, if for every $\in \in A$, $F(\epsilon)=\varnothing$.

Definition $2.3([7])$. For two soft sets $(F, A)$ and $(G, B)$ over a common universe $U$, we say that $(F, A)$ is a soft subset of $(G, B)$ and is denoted by $(F, A) \widetilde{\simeq}(G, B)$ if

i) $A \subset B$ and,

ii) $\forall \epsilon \in A, F(\epsilon)$ and $G(\epsilon)$ are identical approximations.

$(F, A)$ is said to be a soft super set of $(G, B)$, if $(G, B)$ is a soft subset of $(F, A)$.

Definition 2.4 ([7]). Union of two soft sets of $(F, A)$ and $(G, B)$ over the common universe $U$ is the soft set $(\mathrm{H}, \mathrm{C})$, where $\mathrm{C}=\mathrm{A} \cup \mathrm{B}$, and $\forall e \in \mathrm{C}$,

$$
H(e)= \begin{cases}F(e), & \text { if } e \in A-B, \\ G(e), & \text { if } e \in B-A, \\ F(e) \cup G(e), & \text { if } e \in A \cap B .\end{cases}
$$

Definition 2.5 ([9]). Intersection of two soft sets $(F, A)$ and $(G, B)$ over a common universe $U$ is the soft set $(H, C)$, where $C=A \cap B$, and $\forall e \in C, H(e)=F(e) \cap G(e)$. We write $(F, A) \widetilde{\cap}(G, B)=(H, C)$.

Definition $2.6([1])$. The complement of $(F, A)$, denoted by $(F, A)^{c}$, is defined by $(F, A)^{c}=\left(F^{c}, A\right)$, where $\mathrm{F}^{\mathrm{c}}: A \rightarrow \mathrm{P}(\mathrm{U})$ is a function given by $\mathrm{F}^{\mathrm{c}}(\mathrm{x})=\mathrm{U}-\mathrm{F}(\mathrm{x})$ for all $\mathrm{x} \in \mathrm{A}$.

Definition $2.7([2])$. Let $(F, A)$ and $(G, B)$ be two soft sets over $U$, then the Cartesian product of $(F, A)$ and $(G, B)$ is defined as, $(F, A) \times(G, B)=(H, A \times B)$ where $H: A \times B \rightarrow \mathcal{P}(U \times U)$ and $H(a, b)=F(a) \times G(b)$, where $(a, b) \in A \times B$, i.e., $H(a, b)=\left\{\left(h_{i}, h_{j}\right) \mid h_{i} \in F(a), h_{j} \in G(b)\right\}$.

Definition $2.8([2])$. Let $(F, A)$ and $(G, B)$ be two soft sets over $U$, then a soft set relation $R$ from $(F, A)$ to $(G, B)$ is a soft subset of $(F, A) \times(G, B)$. In other words, a soft set relation $R$ from $(F, A)$ to $(G, B)$ is of the form $R=\left(H_{1}, S\right)$ where $S \subset A \times B$ and $H_{1}(a, b)=H(a, b)$ for all $(a, b) \in S$, where $(H, A \times B)=(F, A) \times(G, B)$.

Definition 2.9 ([2]). Let $R$ be a soft set relation on $(F, A)$, then

1. $R$ is reflexive if $H_{1}(a, a) \in R, \forall a \in A$.

2. $R$ is symmetric if $H_{1}(a, b) \in R \Rightarrow H_{1}(b, a) \in R$.

3. $R$ is transitive if $H_{1}(a, b) \in R, H_{1}(b, c) \in R \Rightarrow H_{1}(a, c) \in R$ for every $a, b, c \in A$.

Definition 2.10 ([3]). A binary soft set relation $R$ on $(F, A)$ is an antisymmetric if $F(a) \times F(b) \in R$ and $F(b) \times F(a) \in R$ for every $F(a), F(b) \in(F, A)$ imply $F(a)=F(b)$.

Definition 2.11. [12] Consider a soft set $(F, A)$ equipped with reflexive, transitive soft set relation $\leq$. This relation is called preorder and $(F, A)$ is a preordered soft set.

Definition $2.12([3])$. A binary soft set relation $\leq$ on $(F, A)$ which is reflexive, antisymmetric and transitive is called a partial ordering of $(F, A)$. The triple $(F, A, \leq)$ is called a partially ordered soft set. 
Definition 2.13 ([3]). Let $\leq$ be an ordering of $(F, A)$ and $F(a), F(b)$ be any two elements in $(F, A)$. We say that $F(a)$ and $F(b)$ are comparable in the ordering if $F(a) \leq F(b)$ or $F(b) \leq F(a)$. We say that $F(a)$ and $F(b)$ are incomparable if they are not comparable.

Definition $2.14([3])$. Let $\leq$ be a partially ordering on the soft set $(F, A)$. Then $\leq$ is called a total or linear ordering on $(F, A)$ if every element in $(F, A)$ is comparable in the ordering $\leq$.

Definition 2.15 ([15]). Let $\leq$ be a soft set relation on $(F, A)$, then restriction of a soft set relation $\leq$ to a soft subset $(G, B)$ is defined by:

$$
G(a) \leq_{(G, B)} G(b): \Leftrightarrow F(a) \leq F(b) \text { for all } a, b \in B .
$$

Definition $2.16([14])$. Let $(F, A) \in S(U)$. We say that $\alpha=(p, u)$ is a nonempty soft element of $(F, A)$ if $p \in E$ and $u \in F(p)$. The pair $(p, \varnothing)$, where $p \in E$, will be called an empty soft element of $(F, A)$. Nonempty soft elements of $(F, A)$ and empty soft elements of $(F, A)$ will be called the soft elements of $(F, A)$. The fact that $\alpha$ is a soft element of $(F, A)$ will be denoted by $\alpha \widetilde{\epsilon}(F, A)$.

Remark 2.17. A soft element $\alpha=(p, u)$ of a soft set $(F, A)$ can be considered as a soft set $\left(H_{u}, B\right)$ on $U$ where $B=\{p\} \subseteq E$ and $H_{u}(p)=\{u\} \subseteq U$. Then for a soft set $(F, A)=\{(p, F(p)) \mid p \in A\}$ we can deduce

$$
\{(p, F(p))\}=\widetilde{\bigcup}_{u \in F(p)}\left(H_{\mathfrak{u}},\{p\}\right) .
$$

for $F(p)$ where $p \in A$. Indeed $\widetilde{U}_{\mathfrak{u} \in F(p)}\left(H_{\mathfrak{u}},\{p\}\right)=(K, C)$ where $C=\bigcup\{p\}$ and $K(p)=\cup_{\mathfrak{u} \in F(p)}\{u\}=F(p)$.

Definition 2.18. Let $(F, A)$ be a soft set and $\leq$ be a soft set relation on it. If $(G, B)$ is an another soft set where $B \subseteq A$ and $G(x) \subseteq F(x)$ for all $x$ in $B$ then we can define a soft set relation $\leq_{G \rightarrow F}$ on $(G, B)$ induced from $\leq$ as follows: $(p, u)$ is a soft element of $\leq_{G \rightarrow F}$ where $u \in G(p) \times G(p): \Leftrightarrow(p, u) \widetilde{\epsilon} \leq$ and $(p, u) \widetilde{\epsilon}(G, B) \times(G, B)$.

Example 2.19. Let $U=\left\{u_{1}, u_{2}, u_{3}, u_{4} u_{5}\right\}$ be a universal set and $A=\{a, b, c\}, B=\{a, b\}$ be parameter sets. Let $u$ define soft sets $(F, A)$ and $(G, B)$ as $F(a)=\left\{u_{1}, u_{3}, u_{5}\right\}, F(b)=\left\{u_{2}, u_{4}\right\}, F(c)=\left\{u_{2}, u_{3}, u_{4}\right\}$, and $G(a)=\left\{u_{1}, u_{3}\right\}, G(b)=\left\{u_{2}\right\}$, respectively. Consider the soft set relation $\leq$ on $(F, A)$ as follows

$$
\begin{aligned}
\leq= & F(a) \times F(a), F(b) \times F(b), F(c) \times F(c), F(a) \times F(b)\} \\
=\{ & \{((a, a),(1,1)),((a, a),(1,3)),((a, a),(1,5)),((a, a),(3,1)),((a, a),(3,3)), \\
& ((a, a),(3,5)),((a, a),(5,1)),((a, a),(5,3)),((a, a),(5,5))\},\{((b, b),(2,2)), \\
& ((b, b),(2,4)),((b, b),(4,4)),((b, b),(4,2))\},\{((a, b),(1,2)),((a, b),(1,4)), \\
& ((a, b),(3,2)),((a, b),(3,4)),((a, b),(5,2)),((a, b),(5,4))\}\} .
\end{aligned}
$$

Then we obtain a soft set relation on $(G, B)$ according to $\leq$

$$
\begin{aligned}
& \leq_{(G, B)=}\{\{((a, a),(1,1)),((a, a),(1,3)),((a, a),(3,1)),((a, a),(3,3))\}, \\
&\{((b, b),(2,2))\},\{((a, b),(1,2)),((a, b),(3,2))\}\} \\
&=\{G(a) \times G(a), G(b) \times G(b), G(a) \times G(b)\} .
\end{aligned}
$$

Definition 2.20 ([12]). Let $\leq$ be an ordering of $(F, A)$, and let $(G, B) \widetilde{c}(F, A)$.

- For $a \in A, F(a)$ is a lower bound of $(G, B)$ in the ordered soft set $(F, A, \leq)$ if $F(a) \leq G(x)$ for all $x \in B$.

- For $a \in A, F(a)$ is called infimum of $(G, B)$ in $(F, A, \leq)$ (or the greatest lower bound) if it is the greatest element of the set of all lower bounds of $(G, B)$ in $(F, A, \leq)$.

Similarly,

- For $a \in A, F(a)$ is an upper bound of $(G, B)$ in the ordered soft set $(F, A, \leq)$ if $G(x) \leq F(a)$ for all $x \in \mathrm{B}$. 
- For $a \in A, F(a)$ is called supremum of $(G, B)$ in $(F, A, \leq)$ (or the least upper bound) if it is the least element of the set of all upper bounds of $(G, B)$ in $(F, A, \leq)$.

Definition 2.21 ([12]). Let $(F, A)$ be a preordered soft set. A soft subset $(G, B)$ of $(F, A)$ is directed soft set provided it is nonnull and every finite soft subset of $(G, B)$ has an upper bound in $(G, B)$.

Definition 2.22 ([12]). Let $(F, A)$ be a preordered soft set. We call a nonnull soft subset $(G, B)$ of $(F, A)$ filtered soft set if every finite soft subset of $(G, B)$ has a lower bound.

Definition 2.23. Let $(F, A)$ be a soft set with a preorder soft set relation $\leq$. For $(G, B) \widetilde{c}(F, A)$

i) $\downarrow(G, B)=(H, C)$ where $C=\{a \in A: F(a) \leq G(b)$ for some $b \in B\}$ and $H=\left.F\right|_{C}[12]$.

ii) $\uparrow(G, B)=(K, D)$ where $D=\{a \in A: G(b) \leq F(a)$ for some $b \in B\}$ and $K=\left.F\right|_{D}$ [12].

We also say

iii) $(G, B)$ is a lower soft set iff $(G, B)=\downarrow(G, B)[12]$.

iv) $(G, B)$ is an upper soft set iff $(G, B)=\uparrow(G, B)[12]$.

v) $(G, B)$ is a soft ideal iff it is a directed lower soft set [12].

vi) $(G, B)$ is a soft filter iff it is a filtered upper soft set [17].

Definition 2.24 ([17]). A soft inf-semilattice is a partially ordered soft set $(F, A, \leq)$ in which $F(a), F(b)$ have infimum for any two elements $a, b \in A$.

A soft sup-semilattice is a partially ordered soft set $(F, A)$ in which any $F(a), F(b)$ in $(F, A)$ have a supremum.

A partially ordered soft set $(F, A)$ which is both soft inf-semilattice and soft sup-semilattice is called a soft lattice.

Definition 2.25 ([12]). A posset is said to be directed complete soft sets if every directed soft subset has a supremum.

Definition 2.26 ([14]). Let $(F, A),(G, B) \in S(U)$. A soft relation $T \widetilde{\subseteq}(F, A) \times(G, B)$ is called a soft function from $(F, A)$ to $(G, B)$, which is denoted by $T:(F, A) \widetilde{\rightarrow}(G, B)$, if the following two conditions are satisfied:

SM1 for each soft element $\alpha \widetilde{\epsilon}(F, A)$, there exists only one soft element $\beta \widetilde{\epsilon}(G, B$ ) such that $\alpha T \beta$ (which will be noted as $\mathrm{T}(\alpha)=\beta)$;

SM2 for each empty soft element $\alpha \widetilde{\epsilon}(F, A), T(\alpha)$ is an empty soft element of $(G, B)$.

Note that, from the fact $(F, A)=\widetilde{U}_{p \in A}(p, F(p))=\widetilde{U}_{p \in A} \widetilde{U}_{u \in F(p)}(p,\{u\})$, one can easily check that

$$
\begin{aligned}
T((F, A)) & =T\left(\widetilde{\bigcup}_{p \in A}\{(p, F(p))\}\right)=\widetilde{\bigcup}_{p \in A} T(\{(p, F(p))\})=\widetilde{U}_{p \in A} T\left(\left\{\left(p, \bigcup_{u \in F(p)}\{u\}\right)\right\}\right) \\
& =\widetilde{\bigcup}_{p \in A} T\left(\widetilde{U}_{u \in F(p)}\{(p,\{u\})\}\right)=\widetilde{\bigcup}_{p \in A}\left(\widetilde{U}_{u \in F(p)}\{T(p,\{u\})\}\right) \widetilde{\subseteq}(G, B) .
\end{aligned}
$$

Definition 2.27 ([16]). A soft set function $f:\left(F, A, \leq_{A}\right) \rightarrow\left(G, B, \leq_{B}\right)$ between two partially ordered soft set is called order preserving or monotone iff for all $F(a), F(b)$ in $(F, A), F(a) \leq_{A} F(b) \Rightarrow f(F(a)) \leq_{B} f(F(b))$.

Definition 2.28 ([10]). A soft topology $\widetilde{\tau}$ on a soft set $(F, A)$ is a family of soft subsets of $(F, A)$ satisfying the following properties

i) $\Phi,(F, A) \in \widetilde{\tau}$; 
ii) if $(G, B),(H, C) \in \widetilde{\tau}$, then $(G, B) \widetilde{\cap}(H, C) \in \widetilde{\tau}$;

iii) if $\left(F, A_{\alpha}\right) \in \widetilde{\tau}$ for all $\alpha \in \Lambda$, an index set, then $\widetilde{U}_{\alpha \in \Lambda}\left(F, A_{\alpha}\right) \in \widetilde{\tau}$.

If $\widetilde{\tau}$ is a soft topology on a soft set $(F, A),(F, A, \widetilde{\tau})$ is called the soft topological space.

Definition 2.29 ([10]). If $\widetilde{\tau}$ is a soft topology on $(F, A)$, then the member of $\widetilde{\tau}$ is called soft open set in $(F, A, \widetilde{\tau})$.

Definition $2.30([4])$. Let $(F, A, \widetilde{\tau})$ be a soft topological space and $(G, B) \widetilde{c}(F, A)$. Then, $(G, B)$ is said to be soft closed if the soft set $(G, B)^{c}$ is soft open.

Definition $2.31([4])$. Let $(F, A, \widetilde{\tau})$ be a soft topological space and $(F, B) \widetilde{\subseteq}(F, A)$. Then, the soft interior of $(F, B)$, denoted by $(F, B)^{\circ}$, is defined as the soft union of all soft open subsets of $(F, B)$.

Note that $(F, B)^{\circ}$ is the biggest soft open set that is contained by $(F, B)$.

Definition 2.32 ([10]). A collection $\widetilde{\beta}$ of some soft subsets of $(F, A)$ is called a soft open base or simply a base for some soft topology on $(F, A)$ if the following conditions hold:

i) $\Phi \in \widetilde{\beta}$;

ii) $\widetilde{\cup} \widetilde{\beta}=(F, A)$, i.e., for each $e \in A$ and $x \in F(e)$, there exits $(G, B) \in \widetilde{\beta}$ such that $x \in G(e)$, where $B \subseteq A$;

iii) if $(G, B),(H, C) \in \widetilde{\beta}$ then for each $e \in B \cap C$ and $x \in G(e) \cap H(e)$ there exists $(I, D) \widetilde{\subseteq} \widetilde{\beta}$ such that $(I, D) \widetilde{\subseteq}(G, B) \widetilde{\cap}(H, C)$ and $x \in I(e)$, where $D \subseteq B \cap C$.

Definition $2.33([4])$. Let $(F, A, \widetilde{\tau})$ be a soft topological space and $(F, B) \widetilde{\subseteq}(F, A)$. Then, the soft closure of $(F, B)$, denoted by $\overline{(F, B)}$, is defined as the soft intersection of all soft closed supersets of $(F, B)$.

Note that $\overline{(F, B)}$ is the smallest soft closed set that contains $(F, B)$.

Definition 2.34. We say that a soft topological space $(F, A, \widetilde{\tau})$ is $T_{0}$ soft topological space if for each $\alpha, \beta \widetilde{\epsilon}(F, A), \alpha \neq \beta$, there exists soft open $(G, B)$ such that $\alpha \widetilde{\epsilon}(G, B)$ and $\beta \widetilde{\notin}(G, B)$ or $\beta \widetilde{\epsilon}(G, B)$ and $\alpha \widetilde{\notin}(G, B)$.

Definition $2.35([14])$. Let $(F, A, \widetilde{\tau}),(G, B, \widetilde{v})$ be soft topological spaces and let $T:(F, A) \widetilde{\rightarrow}(G, B)$ be a soft function. We say that $T$ is a soft continuous function (with respect to the soft topologies $\widetilde{\tau}$ and $\widetilde{v}$ ) if for each $(\mathrm{H}, \mathrm{C}) \in \widetilde{v}, \mathrm{~T}^{-1}((\mathrm{H}, \mathrm{C})) \in \widetilde{\tau}$ (i.e., the inverse of a soft open set is a soft open set).

\section{The way-below soft set relation}

We may call meet (Resp. join) operation instead of taking infimum (Resp. supremum) of the elements, and we may use multiplication notation for meet operation.

Definition $3.1([13])$. A soft inf-semilattice $(F, A)$ is called soft meet continuous if it is directed complete soft set and satisfying

$$
F(x) \sup (G, B)=\sup (F(x)(G, B))
$$

for all $x \in A$ and all directed soft subsets $(G, B) \widetilde{C}(F, A)$.

Definition 3.2 ([13]). Let $(F, A, \leq)$ be a partially ordered soft set. We say that $F(a)$ way-below $F(b)$ iff all directed soft subsets $(G, B) \widetilde{\simeq}(F, A)$ for which $\sup (G, B)$ exists, the soft set relation $F(b) \leq \sup (G, B)$ always implies the existence of a $G(d)$ in $(G, B)$ with $F(a) \leq G(d)$. $F(a)$ way-below $F(b)$ is denoted by $F(a) \ll F(b)$.

This definition was expressed simultaneously by Sayed [11] as "Let $(F, A)$ be a posset. For any two elements $F(x), F(y) \in(F, A)$. $F(x)$ is approximate to $F(y)$, and write $F(x) \ll F(y)$, if for any directed soft subset $(G, B) \subset(F, A)$ with $\vee(G, B)$ existing and $F(y) \leq \vee(G, B)$, there exists $G(z) \in(G, B)$ such that $\mathrm{F}(\mathrm{x}) \leq \mathrm{G}(z)$." 
Remark 3.3 ([13]). We write

$$
\begin{aligned}
& \Downarrow F(a)=\{F(b) \mid b \in A \text { with } F(b) \ll F(a)\}, \\
& \uparrow F(a)=\{F(b) \mid b \in A \text { with } F(a) \ll F(b)\} .
\end{aligned}
$$

Definition 3.4 ([13]).

i) A partially ordered soft set $(F, A, R)$ is called soft continuous if it satisfies the axiom of approximation:

$$
(\forall F(a) \in(F, A)) \quad F(a)=\bigvee^{\uparrow} \downarrow F(a),
$$

i.e., for all $F(a)$ in $(F, A)$, the soft set $\downarrow F(a)$ which is $(H, C)$ such that $C=\{b \in A \mid F(b) \ll F(a)\}$ and $H=\left.F\right|_{C}$, is directed and $F(a)=\sup (H, C)$.

ii) A directed complete partially ordered soft set is soft continuous as a partially ordered soft set will be called soft domain.

Theorem 3.5 ([13]). In a partially ordered soft set (F, A) the following statements hold for all $F(a), F(b), F(c), F(d)$ in $(F, A)$.

i) $F(a) \ll F(b) \Rightarrow F(a) \leq F(b)$;

ii) $\mathrm{F}(\mathrm{u}) \leq \mathrm{F}(\mathrm{a}) \ll \mathrm{F}(\mathrm{b}) \leq \mathrm{F}(v) \Rightarrow \mathrm{F}(\mathrm{u}) \ll \mathrm{F}(v)$;

iii) $\mathrm{F}(\mathrm{a}) \ll \mathrm{F}(\mathrm{c})$ and $\mathrm{F}(\mathrm{b}) \ll \mathrm{F}(\mathrm{c}) \Rightarrow \mathrm{F}(\mathrm{a}) \vee \mathrm{F}(\mathrm{b}) \ll \mathrm{F}(\mathrm{c})$ whenever the least upperbound $\mathrm{F}(\mathrm{a}) \vee \mathrm{F}(\mathrm{b})$ exists;

iv) Whenever ( $F, A)$ has a smallest element $\mathrm{F}(0) ; \mathrm{F}(0) \ll \mathrm{F}(\mathrm{a})$.

Theorem 3.6 ([13]).

i) In a partially ordered soft set $(F, A)$, the following conditions are equivalent;

$1 F(a) \ll F(b)$,

$2 \mathrm{~F}(\mathrm{a}) \in(\mathrm{I}, \mathrm{B})$ for every soft ideal $(\mathrm{I}, \mathrm{B})$ of $(\mathrm{F}, \mathrm{A})$ such that $\mathrm{F}(\mathrm{b}) \leq \sup (\mathrm{I}, \mathrm{B})$.

If $(F, A)$ is a soft meet continuous soft semilattice, then conditions (1) and (2) are equivalent to

$3 F(a) \in(I, B)$ for every soft ideal $(I, B)$ of $(F, A)$ such that $F(b)=\sup (I, B)$.

ii) Suppose that there exists a directed soft set $(D, C) \widetilde{\subseteq} \downarrow F(a)$ with $\sup (D, C)=F(a)$. Furthermore, $F(b) \ll F(a)$ if $\mathrm{F}(\mathrm{b}) \ll \mathrm{F}(\mathrm{a})$ in a partially ordered soft set $\downarrow \mathrm{F}(\mathrm{a})$ with the induced order.

Theorem 3.7 ([13]).

i) If $\mathrm{F}(\mathrm{a}) \ll \mathrm{F}(\mathrm{c})$ and $\mathrm{F}(\mathrm{c}) \leq \sup (\mathrm{D}, \mathrm{C})$ for a directed soft set $(\mathrm{D}, \mathrm{C})$ in a continuous partially ordered soft set $(F, A)$, then $F(a) \ll D(e)$ for some $D(e)$ in $(D, C)$.

ii) [11] In a continuous partially ordered soft set (F, A), the way-below soft set relation satisfies the soft interpolation property

$$
F(a) \ll F(c) \Longrightarrow(\exists F(b)) F(a) \ll F(b) \ll F(c) \quad(S I N T)
$$

\section{Soft Scott topology}

Definition $4.1([12])$. Let $(F, A)$ be a directed complete partially ordered soft set and $(G, B) \widetilde{\subseteq}(F, A)$. Then $(G, B)$ is called a Scott soft open set iff the following two conditions are satisfied:

i) $(G, B)=\uparrow(G, B)$;

ii) $\sup (D, C) \in(G, B)$ implies $(D, C) \widetilde{\cap}(G, B) \neq \Phi$ for all directed complete soft sets $(D, C) \widetilde{C}(F, A)$. 
Theorem 4.2 ([12]). The collection of all Scott soft open sets of $(F, A)$ is a soft topology.

Definition 4.3 ([12]). The collection of all Scott soft open sets of $(F, A)$ is called soft Scott topology on $(F, A)$ and this topology will be denoted by $\sigma(F, A)$.

Remark 4.4. We say that a soft subset (G, B) of a directed complete partially ordered soft set $(F, A)$ has the property $(\mathrm{S})$ provided that the following condition is satisfied.

(S) If $\sup (D, C) \in(G, B)$ for any directed soft set $(D, C)$, then there is a $D(c) \in(D, C)$ such that $G(b) \epsilon$ $(G, B)$ for all $D(e) \in(D, C)$ with $G(b) \geq D(e)$.

Definition $4.5([12])$. Let $(F, A, \widetilde{\tau})$ be a soft Scott topology, and $(G, B) \widetilde{\subseteq}(F, A)$. Then $(G, B)$ is called Scott soft closed set if $(G, B)^{\mathrm{C}}$ is Scott open soft set.

Theorem 4.6. In any soft directed complete partially ordered set (FA), we have the following conclusions.

i) a soft set is soft Scott closed iff it is a lower soft set under directed sups;

ii) $\downarrow F(a)=\{F(\bar{a})\}$ for all $F(a)$ in $(F, A)$;

iii) every upper soft set is the soft intersection of Scott soft open neighborhoods;

iv) a soft set is a soft Scott open if and only if it is a soft upper set satisfying $S$.

Proof.

i) $(D, C) \widetilde{\subseteq}(F, A)$ is a lower soft set iff $(F, A)-(D, C)$ is an upper soft set and $(F, A)-(D, C)$ satisfies Definition 4.1 (ii) iff $(D, C)$ is closed under directed sups.

ii) $\downarrow F(a)$ is the smallest lower soft set containing $F(a)$ and closed under directed sups.

iii) Every upper soft set $(G, B)$ is the soft intersection of $(F, A)-\downarrow F(a)$ where $F(a)$ is in $(F, A)-(G, B)$. These sets are soft open by (ii).

iv) $(G, B)$ is soft Scott open if and only if $\uparrow(G, B)=(G, B)$ and satisfying $\mathbf{S}$.

Note that, soft Scott topology is not a $T_{0}$ soft topological space although the classical Scott topology is a $T_{0}$ topological space. This situation is illustrated in the following example.

Example 4.7. Let $U=\left\{u_{1}, u_{2}, u_{3}\right\}$ be a universal set and $E=\left\{p_{1}, p_{2}, p_{3}\right\}$ be a parameter set. Lets define a soft set $(F, A)$ where $A=\left\{p_{2}, p_{3}\right\}$ such that $F\left(p_{2}\right)=\left\{u_{1}\right\}, F\left(p_{3}\right)=\left\{u_{1}, u_{3}\right\}$ and soft set relation $\leq=$ $\left\{F\left(p_{3}\right) \times F\left(p_{3}\right), F_{2}\left(p_{3}\right) \times F\left(p_{2}\right), F\left(p_{2}\right) \times F\left(p_{2}\right)\right\}$. Then, soft Scott topology is $\widetilde{\tau}=\left\{\Phi,(F, A),\left\{F\left(p_{2}\right)\right\}\right\}$, which is not a $T_{0}$ soft topological space since for $\alpha=\left(p_{3}, u_{1}\right)$ and $\beta=\left(p_{3}, u_{3}\right)$ are different soft elements but there is no soft open $(G, B)$ satisfying $\alpha \widetilde{\epsilon}(G, B)$ and $\beta \widetilde{\notin}(G, B)$ or $\beta \widetilde{\epsilon}(G, B)$ and $\alpha \widetilde{\notin}(G, B)$.

Theorem 4.8. If $(F, A)$ is a soft domain, then all $\uparrow F(a)$ for $F(a)$ in $(F, A)$ are soft Scott open. Conversely, if $(F, A)$ is a directed complete partially ordered soft set and $F(b) \in \operatorname{int}(\uparrow F(a))$ then $F(a) \ll F(b)$.

Proof. Let $(D, C)$ be a soft directed set with $\sup (D, C) \in \uparrow F(a)$. Then Theorem 3.7 implies the existence of $D(c)$ in $(D, C)$ such that $F(a) \ll D(c)$. Hence $\uparrow F(a)$ is open by Definition 4.1.

Suppose $(F, A)$ is a directed complete partially ordered soft set and $F(b) \in \operatorname{int}(\uparrow F(a))$. If $(D, C)$ is a soft directed set with $F(b) \leq \sup (D, C)$, then $\sup (D, C) \in \operatorname{int}(\uparrow F(a))$ by Definition 4.1 and hence $D(c) \in \operatorname{int}(\uparrow F(a))$ for some $D(c)$ in $(D, C)$ by Definition 4.1. Thus $F(a) \leq D(c)$ then $F(a) \ll F(b)$.

Theorem 4.9. Let $(\mathrm{F}, \mathrm{A})$ be a soft domain. 
i) An upper soft set $(\mathrm{G}, \mathrm{B})$ is soft Scott open iff for every $\mathrm{G}(\mathrm{b})$ in $(\mathrm{G}, \mathrm{B})$ there is $\mathrm{G}\left(\mathrm{b}^{\prime}\right)$ in $(\mathrm{G}, \mathrm{B})$ such that $\mathrm{G}\left(\mathrm{b}^{\prime}\right) \ll \mathrm{G}(\mathrm{b})$.

ii) The soft sets of the form $\uparrow F(a), F(a)$ in $(F, A)$, form a soft basis for soft Scott topology.

iii) With respect to $\sigma(F, A)$, int $(\uparrow F(a))=\uparrow F(a)$.

Proof.

i) Let $(G, B)$ be a soft Scott open and $G(b)$ in $(G, B)$. As in a soft domain $\downarrow G(b)$ is a soft directed and $G(b)=\sup \downarrow G(b)$. We conclude that there is $G\left(b^{\prime}\right) \ll G(b)$ with $G\left(b^{\prime}\right)$ in $(G, B)$ by Definition 4.1. If conversely for every $G(b)$ in $(G, B)$ there is $G\left(b^{\prime}\right)$ in $(G, B)$ such that $(G, B)$ is the soft union of $\uparrow G\left(b^{\prime}\right), G\left(b^{\prime}\right)$ in $(G, B)$ which is soft Scott open by Theorem 4.8, hence $(G, B)$ is Scott open.

ii) This follows directly from (i).

iii) If $G(b)$ is $\operatorname{in} \operatorname{int}(\uparrow F(a))$, then by (i) there is $G(c)$ in $\uparrow F(a), G(c) \ll G(b)$. Then $G(b)$ is in $\uparrow F(a)$. Obviously $\uparrow F(a) \widetilde{\subseteq} \operatorname{int}(\uparrow F(a))$.

\section{Soft Scott continuous functions}

Theorem 5.1. For a soft function $\mathrm{T}$ from a directed complete partially ordered soft set $(\mathrm{F}, \mathrm{A})$ into directed complete partially ordered soft set (G,B). Then the followings are equivalent:

i) $T$ is soft continuous function with respect to the soft Scott topology, i.e.,

$$
\mathrm{T}^{-1}((\mathrm{D}, \mathrm{C})) \in \sigma((\mathrm{F}, \mathrm{A})) \text { for all }(\mathrm{D}, \mathrm{C}) \in \sigma((\mathrm{G}, \mathrm{B})) .
$$

ii) $\mathrm{T}$ preserves suprema of directed soft sets, i.e., $\mathrm{T}$ is order preserving and $\mathrm{T}(\sup (\mathrm{D}, \mathrm{C}))=\sup \mathrm{T}((\mathrm{D}, \mathrm{C}))$, for all directed soft subsets $(D, C)$ of $(F, A)$.

If $(F, A)$ and $(G, B)$ are soft domains, then $i$, ii are equivalent to each of the following two conditions:

iii) $G(b) \ll T(F(a))$ iff for some $F\left(a^{\prime}\right) \ll F(a)$ one has $G(b) \ll T\left(F\left(a^{\prime}\right)\right)$ for all $F(a)$ in $(F, A)$ and $G(b)$ in $(G, B)$.

iv) $T(F(a))=\sup \left\{T\left(F\left(a^{\prime}\right)\right): F\left(a^{\prime}\right) \ll F(a)\right\}$ for all $F(a)$ in $(F, A)$.

Proof.

$\mathbf{i} \Rightarrow$ ii We need to show $\mathrm{T}$ is order preserving.

Suppose $T(F(a)) \nless T\left(F\left(a^{\prime}\right)\right)$, then soft Scott open set $(H, C)=(F, A)-\downarrow T\left(F\left(a^{\prime}\right)\right)$ contains $T(F(a))$. Thus $(D, C)=T^{-1}((H, C))$ is soft Scott open set which contains $F(a)$ not containing $F\left(a^{\prime}\right)$. But then $F(a) \not F\left(a^{\prime}\right)$ as $(D, C)$ is a soft upper set. Thus $F(a) \leq F\left(a^{\prime}\right)$ implies $T(F(a)) \leq T\left(F\left(a^{\prime}\right)\right)$. Now we need to show $T(\sup (D, C))=\sup T((D, C))$. Let $(D, C)$ be directed soft subset of $(F, A)$. Then $T((D, C))$ is directed and $\sup T((D, C)) \leq T(\sup (D, C))$, since $T$ is order preserving. Set $F(a)=\sup (D, C)$ and $G(b)=\sup T((D, C))$. We claim $T(F(a)) \leq G(b)$. Suppose $T(F(a)) \nless G(b)$. The soft Scott open set $(G, B)-\downarrow G(b)$ contains $T(F(a))$; thus $T^{-1}((G, B)-\downarrow G(b))$ is a soft Scott open set which contains $F(a)$. It follows that there is $D(c)$ in $(D, C)$ such that $D(c)$ is in $T^{-1}((G, B)-\downarrow G(b))$. Then $T(D(c))$ is in $(G, B)-\downarrow G(b)$, i.e., $T(D(c)) \not G(b)=\sup T((D, C))$. This contradiction proves our claim.

$\mathbf{i i} \Rightarrow \mathbf{i}$ Let $(H, C)$ be a soft Scott subset of $(G, B)$. In order to show $T^{-1}((H, C))$ is soft Scott closed in $(F, A)$ we take a soft directed subset $(D, C)$ of $T^{-1}((H, C))$. Then by ii since $T(\sup (D, C))=\sup T((D, C))$. But sup $T((D, C))$ is in $(H, C)$ by Theorem 4.6 since $(H, C)$ is soft Scott closed and $T((D, C))$ is directed owing to the monotocity of $T$. Then $T(\sup (D, C))$ is in $(H, C)$ and hence $\sup (D, C)$ is in $\mathrm{T}^{-1}((\mathrm{H}, \mathrm{C}))$. Then $\mathrm{T}^{-1}((\mathrm{H}, \mathrm{C}))$ is soft Scott closed by Theorem 4.6. 
ii $\Rightarrow$ iv Since $\downarrow F(a)$ is directed and $F(a)=\sup \downarrow F(a)$.

iv $\Rightarrow$ iii From iv we can conclude that $T$ is monotone. Indeed if $F\left(a^{\prime}\right) \leq F(a)$, then $\downarrow F\left(a^{\prime}\right) \subseteq \downarrow F(a)$ and consequently $T\left(F\left(a^{\prime}\right)\right)=\sup T\left(\downarrow F\left(a^{\prime}\right)\right) \leq \sup T(\downarrow F(a))=F(a)$. Now let $G(b) \ll T\left(F\left(a^{\prime}\right)\right)=\sup T(\downarrow$ $\left.F\left(a^{\prime}\right)\right)$; since $T$ is monotone, $T\left(\downarrow F\left(a^{\prime}\right)\right)$ is directed. Thus by Theorem 3.7 there is a $F\left(a^{\prime \prime}\right) \ll F\left(a^{\prime}\right)$ with $G(b) \ll T\left(F\left(a^{\prime \prime}\right)\right)$. Conversely, if $G(b) \ll T\left(F\left(a^{\prime \prime}\right)\right)$ for some $F\left(a^{\prime \prime}\right) \ll F\left(a^{\prime}\right)$, then $G(b) \ll$ $\mathrm{T}\left(\mathrm{F}\left(\mathrm{a}^{\prime}\right)\right)$ by monotocity of $\mathrm{T}$ and Definition 4.1 for $\ll$.

iii $\Rightarrow$ i Let $(D, C) \in \sigma((G, B))$ and $F(a) \in T^{-1}((D, C))$. By Theorem 4.9 there is $G(b)$ in $(D, C)$ with $G(b) \ll T(F(a))$. By iii we find $F\left(a^{\prime}\right) \ll F(a)$ such that $G(b) \ll T\left(F\left(a^{\prime}\right)\right)$; we will finish the proof if we show $T\left(\uparrow F\left(a^{\prime}\right)\right) \subseteq(D, C)$. Now take $F\left(a^{\prime \prime}\right)$ with $F\left(a^{\prime}\right) \ll F\left(a^{\prime \prime}\right)$. For every $G\left(b^{\prime}\right) \ll T\left(F\left(a^{\prime}\right)\right)$ we have $G\left(b^{\prime}\right) \ll T\left(F\left(a^{\prime \prime}\right)\right)$ by iii; and consequently $T\left(F\left(a^{\prime}\right)\right)=\sup \downarrow T\left(F\left(a^{\prime}\right)\right) \leq T\left(F\left(a^{\prime \prime}\right)\right)$. But $\mathrm{G}(\mathrm{b}) \leq \mathrm{T}\left(\mathrm{F}\left(\mathrm{a}^{\prime}\right)\right)$ by Theorem 3.5 and $\mathrm{G}(\mathrm{b})$ in $(\mathrm{D}, \mathrm{C})$; hence $\mathrm{T}\left(\mathrm{F}\left(\mathrm{a}^{\prime \prime}\right)\right) \in(\mathrm{D}, \mathrm{C})$ by Definition 4.1.

Definition 5.2. A soft function $T:(F, A) \widetilde{\rightarrow}(G, B)$ between directed partially ordered soft sets is soft Scott continuous if and only if it satisfies the equivalent conditions in Theorem 5.1 (i), (ii), (iii).

Example 5.3. Let $U=\left\{u_{1}, u_{2}, u_{3}\right\}$ be a universal set and $E=\left\{p_{1}, p_{2}, p_{3}\right\}$ be a parameter set. Let us define a soft set $\left(F_{1}, A_{1}\right)$ where $A_{1}=\left\{p_{1}\right\}$ such that $F_{1}\left(p_{1}\right)=\left\{u_{1}, u_{2}\right\}$ and a soft set $\left(F_{2}, A_{2}\right)$ where $A_{2}=\left\{p_{2}, p_{3}\right\}$ such that $F_{2}\left(p_{2}\right)=\left\{u_{1}\right\}, F_{2}\left(p_{3}\right)=\left\{u_{1}, u_{3}\right\}$ and soft set relations $\leq_{1}=\left\{F_{1}\left(p_{1}\right) \times F_{1}\left(p_{1}\right)\right\}$ and $\leq_{2}=\left\{F_{2}\left(p_{3}\right) \times\right.$ $\left.\mathrm{F}_{2}\left(\mathrm{p}_{3}\right), \mathrm{F}_{2}\left(\mathrm{p}_{3}\right) \times \mathrm{F}_{2}\left(\mathrm{p}_{2}\right), \mathrm{F}_{2}\left(\mathrm{p}_{2}\right) \times \mathrm{F}_{2}\left(\mathrm{p}_{2}\right)\right\}$, soft topologies $\widetilde{\tau}_{1}=\left\{\Phi,\left(\mathrm{F}_{1}, A_{1}\right)\right\}, \widetilde{\tau}_{2}=\left\{\Phi,\left(\mathrm{F}_{2}, A_{2}\right),\left\{\mathrm{F}\left(\mathrm{p}_{2}\right)\right\}\right\}$, respectively. A soft function $T$ can be defined as follows:

$$
\mathrm{T}\left(\mathrm{p}_{1}, \varnothing\right)=\left(\mathrm{p}_{1}, \varnothing\right) ; \mathrm{T}\left(\mathrm{p}_{1},\left\{\mathrm{u}_{1}\right\}\right)=\left(\mathrm{p}_{2},\left\{\mathrm{u}_{1}\right\}\right) ; \mathrm{T}\left(\mathrm{p}_{1},\left\{\mathrm{u}_{2}\right\}\right)=\left(\mathrm{p}_{3},\left\{\mathrm{u}_{3}\right\}\right) .
$$

Since $T^{-1}\left(F_{2}, A_{2}\right)=\left(F_{1}, A_{1}\right) \in \widetilde{\tau}_{1}$ but $T^{-2}\left(\left\{F_{2}\left(p_{2}\right)\right\}\right)=\left\{p_{1},\left\{u_{1}\right\}\right\}$ is not in $\widetilde{\tau_{1}}, T$ is not continuous.

Example 5.4. Let $U=\left\{u_{1}, u_{2}, u_{3}\right\}$ be an initial universe, $A=\left\{a_{1}, a_{2}\right\}$ and $B=\left\{b_{1}, b_{2}, b_{3}\right\}$ be parameter sets and let define soft sets $(F, A)=\left\{\left(a_{1},\left\{u_{2}\right\}\right),\left(a_{2},\left\{u_{1}, u_{3}\right\}\right)\right\}$ and $(G, B)=\left\{\left(b_{1},\left\{u_{1}\right\}\right),\left(b_{2},\left\{u_{3}\right\}\right)\right.$, $\left.\left(b_{3},\left\{u_{2}, u_{3}\right\}\right)\right\}$. Consider soft set relations on $(F, A)$ and $(G, B), s_{1}=\left\{F\left(a_{1}\right) \times F\left(a_{1}\right), F\left(a_{1}\right) \times F\left(a_{2}\right), F\left(a_{1}\right) \times\right.$ $\left.F\left(a_{2}\right)\right\}$ and $\leq_{2}=\left\{G\left(b_{1}\right) \times G\left(b_{1}\right), G\left(b_{2}\right) \times G\left(b_{2}\right), G\left(b_{3}\right) \times G\left(b_{3}\right), G\left(b_{3}\right) \times G\left(b_{1}\right), G\left(b_{1}\right) \times G\left(b_{2}\right), G\left(b_{3}\right) \times G\left(b_{2}\right)\right\}$, respectively and soft Scott topologies $\tau_{1}=\left\{(F, A),\left\{F\left(a_{2}\right)\right\}, \Phi\right\}$ and $\tau_{2}=\left\{(G, B), \Phi,\left\{G\left(b_{2}\right)\right\},\left\{G\left(b_{2}\right)\right.\right.$, $\left.\left.G\left(b_{1}\right)\right\}\right\}$ on $(F, A)$ and $(G, B)$, respectively. Now let us define soft function $T:(F, A) \widetilde{\rightarrow}(G, B)$ as follows:

$$
T\left(a_{1}, u_{2}\right)=\left(b_{3}, u_{2}\right) ; T\left(a_{2}, u_{1}\right)=\left(b_{2}, u_{3}\right) ; T\left(a_{2}, u_{3}\right)=\left(b_{2}, u_{3}\right) .
$$

Continuity of the soft function T can be easily checked by part (i) of Theorem 5.1 as

$$
\mathrm{T}^{-1}(\Phi)=\Phi ; \mathrm{T}^{-1}(\mathrm{G}, \mathrm{B})=(\mathrm{F}, \mathrm{A}) ; \mathrm{T}^{-1}\left(\left\{\mathrm{G}\left(\mathrm{b}_{2}\right)\right\}\right)=\left\{\mathrm{F}\left(\mathrm{a}_{2}\right) ; \mathrm{T}^{-1}\left(\left\{\mathrm{G}\left(\mathrm{b}_{2}\right), \mathrm{G}\left(\mathrm{b}_{1}\right)\right\}\right)=\left\{\mathrm{F}\left(\mathrm{a}_{2}\right)\right\},\right.
$$

so $\mathrm{T}$ is a continuous soft function, since it satisfies Theorem 5.1 (i).

Also part (ii) of Theorem 5.1 can be checked simultaneously by finding all directed soft set $(F, A)$; $\left\{F\left(a_{2}\right)\right\},\left\{F\left(a_{1}\right)\right\}$; in $(F, A)$ and examining their supremums images under the soft function $T$ as

- $T(\sup (F, A))=T\left(\left\{F\left(a_{2}\right)\right\}\right)=G\left(b_{2}\right)=\sup T(F, A)$,

- $T\left(\sup \left\{F\left(a_{2}\right)\right\}\right)=T\left(F\left(a_{2}\right)\right)=G\left(b_{2}\right)=\sup T\left(\left\{F\left(a_{2}\right)\right\}\right)$,

- $T\left(\sup \left\{F\left(a_{1}\right)\right\}\right)=T\left(F\left(a_{1}\right)\right)=\left(b_{3},\left\{u_{2}\right\}\right)=\sup T\left(\left\{F\left(a_{1}\right)\right\}\right)$.

Thus $\mathrm{T}$ is soft Scott continuous by Theorem 5.1.

Example 5.5. Let $\mathrm{U}=\left\{\mathrm{u}_{1}, \mathrm{u}_{2}, \mathrm{u}_{3}, \mathrm{u}_{4}\right\}$ and $\mathrm{V}=\left\{v_{1}, v_{2}, v_{3}\right\}$ be the initial universes, $A=\left\{\mathrm{a}_{1}, \mathrm{a}_{2}, \mathrm{a}_{3}\right\}$ and $B=\left\{b_{1}, b_{2}, b_{3}, b_{4}\right\}$ be parameter sets and let define soft sets $(F, A)=\left\{\left(a_{1},\left\{u_{2}, u_{3}\right\}\right),\left(a_{2},\left\{u_{1}, u_{4}\right\}\right),\left(a_{3}\right.\right.$, $\left.\left.\left\{\mathrm{u}_{1}, \mathrm{u}_{3}, \mathrm{u}_{4}\right\}\right)\right\}$ and $(\mathrm{G}, \mathrm{B})=\left\{\left(\mathrm{b}_{1},\left\{v_{3}\right\}\right),\left(\mathrm{b}_{2},\left\{v_{1}, v_{3}\right\}\right),\left(\mathrm{b}_{3},\left\{v_{2}\right\}\right),\left(\mathrm{b}_{4},\left\{v_{1}, v_{2}\right\}\right)\right\}$. Consider soft set relations on 
$(F, A)$ and $(G, B)$, by $\leq_{(F, A)}=\left\{F\left(a_{1}\right) \times F\left(a_{1}\right), F\left(a_{2}\right) \times F\left(a_{2}\right), F\left(a_{3}\right) \times F\left(a_{3}\right), F\left(a_{3}\right) \times F\left(a_{2}\right), F\left(a_{1}\right) \times F\left(a_{2}\right)\right\}$ and $\leq_{(G, B)}=\left\{G\left(b_{1}\right) \times G\left(b_{1}\right), G\left(b_{2}\right) \times G\left(b_{2}\right), G\left(b_{3}\right) \times G\left(b_{3}\right), G\left(b_{4}\right) \times G\left(b_{4}\right), G\left(b_{1}\right) \times G\left(b_{2}\right), G\left(b_{2}\right) \times G\left(b_{4}\right), G\left(b_{1}\right) \times\right.$ $\left.\mathrm{G}\left(\mathrm{b}_{4}\right)\right\}$, respectively and soft Scott topologies $\tau_{1}=\left\{(\mathrm{F}, \mathrm{A}),\left\{\mathrm{F}\left(\mathrm{a}_{2}\right), \mathrm{F}\left(\mathrm{a}_{3}\right)\right\},\left\{\mathrm{F}\left(\mathrm{a}_{1}\right), \mathrm{F}\left(\mathrm{a}_{2}\right)\right\},\left\{\mathrm{F}\left(\mathrm{a}_{2}\right)\right\}, \Phi\right\}$ and $\tau_{2}=\left\{(G, B), \Phi,\left\{G\left(b_{4}\right)\right\},\left\{G\left(b_{1}\right), G\left(b_{2}\right), G\left(b_{4}\right)\right\},\left\{G\left(b_{2}\right), G\left(b_{4}\right)\right\}\right\}$ on $(F, A)$ and $(G, B)$, respectively. Now let us define soft function $T:(F, A) \widetilde{\rightarrow}(G, B)$ as follows:

$$
\begin{aligned}
& T\left(a_{1}, u_{2}\right)=\left(b_{1}, v_{3}\right) ; T\left(a_{1}, u_{3}\right)=\left(b_{1}, v_{3}\right) ; T\left(a_{2}, u_{1}\right)=\left(b_{4}, v_{2}\right) T\left(a_{2}, u_{1}\right)=\left(b_{4}, v_{2}\right), \\
& T\left(a_{2}, u_{4}\right)=\left(b_{4}, v_{2}\right), T\left(a_{3}, u_{1}\right)=\left(b_{2}, v_{1}\right), T\left(a_{3}, u_{3}\right)=\left(b_{2}, v_{3}\right), T\left(a_{3}, u_{4}\right)=\left(b_{2}, v_{3}\right) .
\end{aligned}
$$

Continuity of the soft function T can be easily checked by part (i) of Theorem 5.1 as

$$
\begin{aligned}
\mathrm{T}^{-1}(\Phi) & =\Phi ; \mathrm{T}^{-1}(\mathrm{G}, \mathrm{B})=(\mathrm{F}, \mathrm{A}) ; \mathrm{T}^{-1}\left(\left\{\mathrm{G}\left(\mathrm{b}_{1}\right), \mathrm{G}\left(\mathrm{b}_{2}\right), \mathrm{G}\left(\mathrm{b}_{4}\right)\right\}\right)=(\mathrm{F}, \mathrm{A}), \\
\mathrm{T}^{-1}\left(\left\{\mathrm{G}\left(\mathrm{b}_{2}\right), \mathrm{G}\left(\mathrm{b}_{4}\right)\right\}\right) & =\left\{\mathrm{F}\left(\mathrm{a}_{2}\right), \mathrm{F}\left(\mathrm{A}_{3}\right)\right\} \mathrm{T}^{-1}\left(\left\{\mathrm{G}\left(\mathrm{b}_{4}\right)\right\}\right)=\left\{\mathrm{F}\left(\mathrm{a}_{2}\right)\right\} .
\end{aligned}
$$

So $T$ is a continuous soft function, since it satisfies Theorem 5.1 (i).

Also part (ii) of Theorem 5.1 can be checked simultaneously by finding all directed soft sets $(F, A)$; $\left\{F\left(a_{2}\right), F\left(a_{3}\right)\right\},\left\{F\left(a_{1}\right), F\left(a_{2}\right)\right\},\left\{F\left(a_{2}\right)\right\},\left\{F\left(a_{3}\right)\right\},\left\{F\left(a_{1}\right)\right\} ;$ in $(F, A)$ and let us examine their supremums images under the soft function $\mathrm{T}$ as followings

- $T(\sup (F, A))=T\left(\left\{F\left(a_{2}\right)\right\}\right)=G\left(b_{2}\right)=\sup T(F, A)$.

- $\sup T\left(\left\{F\left(a_{2}\right), F\left(a_{3}\right)\right\}\right)=\sup \left\{\left(b_{2}, v_{1}\right),\left(b_{2}, v_{3}\right),\left(b_{4}, v_{2}\right)\right\}$.

To find $\sup \left\{\left(b_{2}, v_{1}\right),\left(b_{2}, v_{3}\right),\left(b_{4}, v_{2}\right)\right\}$ we need to define $\left(G_{1}^{\prime},\left\{b_{2}, b_{4}\right\}\right)$ by $G_{1}^{\prime}\left(b_{2}\right)=\left\{v_{1}, v_{2}\right\}$ and $\mathrm{G}_{1}^{\prime}\left(\mathrm{b}_{4}\right)=\left\{v_{2}\right\}$, then by using Definition 2.18, soft set relation $\leq_{\mathrm{G}_{1}^{\prime}}=\left\{\left\{\left(\left(\mathrm{b}_{2}, \mathrm{~b}_{2}\right),\left(v_{1}, v_{1}\right)\right),\left(\left(\mathrm{b}_{2}, \mathrm{~b}_{2}\right)\right.\right.\right.$, $\left.\left.\left(v_{1}, v_{2}\right)\right),\left(\left(b_{2}, b_{2}\right),\left(v_{2}, v_{1}\right)\right),\left(\left(b_{2}, b_{2}\right),\left(v_{2}, v_{2}\right)\right)\right\},\left\{\left(\left(b_{4}, b_{4}\right),\left(v_{2}, v_{2}\right)\right)\right\},\left\{\left(\left(b_{2}, b_{4}\right),\left(v_{1}, v_{2}\right)\right),\left(\left(b_{2}, b_{1}\right),\left(v_{2}\right.\right.\right.$, $\left.\left.\left.\left.v_{2}\right)\right)\right\}\right\}=\left\{\mathrm{G}_{1}^{\prime}\left(\mathrm{b}_{2}\right) \times \mathrm{G}_{1}^{\prime}\left(\mathrm{b}_{2}\right), \mathrm{G}_{1}^{\prime}\left(\mathrm{b}_{4}\right) \times \mathrm{G}_{1}^{\prime}\left(\mathrm{b}_{4}\right), \mathrm{G}_{1}^{\prime}\left(\mathrm{b}_{2}\right) \times \mathrm{G}_{1}^{\prime}\left(\mathrm{b}_{4}\right)\right\}$, thus $\sup \left\{\left(\mathrm{b}_{2}, v_{1}\right),\left(\mathrm{b}_{2}, v_{3}\right),\left(\mathrm{b}_{4}, v_{2}\right)\right\}=$ $\left(b_{4},\left\{v_{2}\right\}\right)$ can be obtained. Also $T\left(\sup \left\{F\left(a_{3}\right), F\left(a_{2}\right)\right\}\right)=T\left(F\left(a_{2}\right)\right)=\left\{b_{4},\left\{v_{2}\right\}\right\}$ can be found. Hence we show that $\sup \left\{\left(b_{2}, v_{1}\right),\left(b_{2}, v_{3}\right),\left(b_{4}, v_{2}\right)\right\}$ and $T\left(\sup \left\{F\left(a_{3}\right), F\left(a_{2}\right)\right\}\right)$ are equal.

- Let us find $\sup T\left\{F\left(a_{1}\right), F\left(a_{2}\right)\right\}=\sup \left\{\left(b_{1}, v_{3}\right),\left(b_{4}, v_{2}\right)\right\}$.

To find $\sup \left\{\left(b_{1}, v_{3}\right),\left(b_{4}, v_{2}\right)\right\}$ we need to define $\left(G_{2},\left\{b_{1}, b_{4}\right\}\right)$ by $G_{2}\left(b_{1}\right)=\left\{v_{3}\right\}$ and $G_{2}\left(b_{4}\right)=$ $\left\{v_{2}\right\}$ then by using Definition 2.18 soft set relation $\leq_{G_{2}}=\left\{\left\{\left(\left(b_{1}, b_{1}\right),\left(v_{3}, v_{3}\right)\right),\left(\left(b_{4}, b_{4}\right),\left(v_{2}, v_{2}\right)\right)\right.\right.$, $\left.\left.\left\{\left(\left(b_{1}, b_{4}\right),\left(v_{3}, v_{2}\right)\right),\right\}\right\}\right\}$, then $\sup \left\{\left(b_{1}, v_{3}\right),\left(b_{4}, v_{2}\right)\right\}=\left(b_{4}, v_{2}\right)$. Also $T\left(\sup \left\{F\left(a_{1}\right), F\left(a_{2}\right)\right\}\right)=T\left(F\left(a_{2}\right)\right)=$ $\left(b_{4}, v_{2}\right)$ which is equal to $\sup \left\{\left(b_{1}, v_{3}\right),\left(b_{4}, v_{2}\right)\right\}$.

- Let us find $\sup T\left\{F\left(a_{2}\right)\right\}=\sup \left\{\left(b_{4}, v_{2}\right)\right\}$.

To find $\sup \left\{\left(b_{4}, v_{2}\right)\right\}$ we need to define $\left(G_{3},\left\{b_{4}\right\}\right)$ by $G_{3}\left(b_{4}\right)=\left\{v_{2}\right\}$ then by using Definition 2.18 soft set relation $\leq_{\mathrm{G}_{3}}=\left\{\left\{\left(\left(\mathrm{b}_{4}, \mathrm{~b}_{4}\right),\left(v_{2}, v_{2}\right)\right)\right\}\right.$ then $\sup \left\{\left(\mathrm{b}_{4}, v_{2}\right)\right\}=\left(\mathrm{b}_{4}, v_{2}\right)$. Also, $\mathrm{T}\left(\sup \left\{\mathrm{f}\left(\mathrm{a}_{2}\right)\right\}\right)=$ $\mathrm{T}\left(\mathrm{F}\left(\mathrm{a}_{2}\right)\right)=\left(\mathrm{b}_{4}, v_{2}\right)$ which is equal to $\sup \left\{\left(\mathrm{b}_{4}, v_{2}\right)\right\}$.

- Let us find $\sup T\left\{F\left(a_{3}\right)\right\}=\sup \left\{\left(b_{2}, v_{1}\right),\left(b_{2}, v_{3}\right)\right\}$.

To find $\sup \left\{\left(b_{2}, v_{1}\right),\left(b_{2}, v_{3}\right)\right\}$ we need to define $G_{4},\left\{b_{2}\right\}$ by $G_{4}\left(b_{2}\right)=\left\{v_{1}, v_{3}\right\}$ then by using Definition 2.18 soft set relation $\leq_{G_{4}}=\left\{\left\{\left(\left(b_{2}, b_{2}\right),\left(v_{1}, v_{1}\right)\right),\left(\left(b_{2}, b_{2}\right),\left(v_{1}, v_{3}\right)\right),\left(\left(b_{2}, b_{2}\right),\left(v_{3}, v_{1}\right)\right),\left(\left(b_{2}, b_{2}\right),\left(v_{3}, v_{3}\right)\right)\right\}\right.$ then $\sup \left\{\left(b_{2}, v_{1}\right),\left(b_{2}, v_{3}\right)\right\}=\left\{\left(b_{2}, v_{1}\right),\left(b_{2}, v_{3}\right)\right\}=G_{4}\left(b_{2}\right)$. Also $T\left(\sup \left\{F\left(a_{3}\right)\right\}\right)=T\left(F\left(a_{3}\right)\right)=\left\{\left(b_{2}, v_{1}\right)\right.$, $\left.\left(b_{2}, v_{3}\right)\right\}$, thus $T\left(\sup \left\{F\left(a_{3}\right)\right\}\right)=\sup \left\{\left(b_{2}, v_{1}\right),\left(b_{2}, v_{3}\right)\right\}$.

- Let us find $\sup T\left\{F\left(a_{1}\right)\right\}=\sup \left\{\left(b_{1}, v_{3}\right)\right\}$.

To find $\sup \left\{\left(b_{1}, v_{3}\right)\right\}$ we need to define $\left(G_{5},\left\{b_{1}\right\}\right)$ by $G_{5}\left(b_{1}\right)=\left\{v_{3}\right\}$ then by using Definition 2.18 soft set relation $\leq_{G_{5}}=\left\{\left\{\left(\left(b_{1}, b_{1}\right),\left(v_{3}, v_{3}\right)\right)\right\}\right.$ then $\sup \left\{\left(b_{1}, v_{3}\right)\right\}=\left(b_{1}, v_{3}\right)$. Also, $T\left(\sup \left\{F\left(a_{1}\right)\right\}\right)=$ $\mathrm{T}\left(\mathrm{F}\left(\mathrm{a}_{1}\right)\right)=\left(\mathrm{b}_{1}, v_{3}\right)$ which is equal to $\sup \left\{\left(\mathrm{b}_{1}, v_{3}\right)\right\}$.

Thus $T$ is soft Scott continuous by Theorem 5.1. 
Theorem 5.6 (Least fixed point theorem for soft Scott Continuous functions). Let ( $\mathrm{F}, \mathrm{A})$ be a directed complete partially ordered soft set with a least element $F\left(a_{0}\right)$.

i) Existence: Every soft Scott continuous self continuous $T:(F, A) \rightarrow(F, A)$ has a least fixed point $\operatorname{LFP}(T)$.

ii) Construction: The least fixed-point can be approximated by recursively defined Kleene chain:

$$
K_{0}=F\left(a_{0}\right), K_{n+1}=T\left(K_{n}\right)=T^{n+1}\left(K_{0}\right)
$$

in the sense that

$$
\operatorname{LFP}(T)=\sup _{n} K_{n}=\sup _{n} T^{n}\left(K_{0}\right) .
$$

iii) Preservation: Let (G, B) be a second directed complete partially ordered soft set with bottom and let

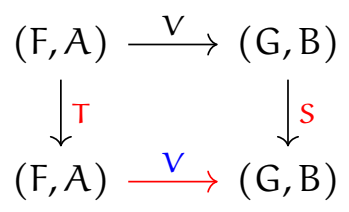

be a commuting diagram of soft Scott continuous maps. Then $\mathrm{V}(\operatorname{LFP}(\mathrm{T}))=\operatorname{LFP}\left(\left.\mathrm{S}\right|_{\uparrow \vee}\left(\mathrm{F}_{0}\right)\right)$.

Proof. $\mathrm{K}_{0}=\mathrm{F}\left(\mathrm{a}_{0}\right)=\mathrm{T}\left(\mathrm{F}\left(\mathrm{a}_{0}\right)\right)=\mathrm{K}_{1}$, since $\mathrm{T}$ is order preserving we have $\mathrm{K}_{1}=\mathrm{T}\left(\mathrm{K}_{0}\right) \leq \mathrm{T}\left(\mathrm{K}_{1}\right)=\mathrm{K}_{2}$ and by induction we can obtain $T\left(K_{n}\right) \leq T\left(K_{n+1}\right)$ for all $n$. As $K_{n}$ is increasing, it has a least upper bound $F(a)=\sup _{n} K_{n}$ in $(F, A)$. By the continuity of $T$, we have $T(F(a))=T\left(\sup _{n} K_{n}\right)=\sup _{n} K_{n+1}=F(a)$. Thus $F(a)$ is a fixed point of $T$. Actually it is the smallest fixed point of $T$. Indeed, let $F\left(a^{\prime}\right)=T\left(F\left(a^{\prime}\right)\right)$ be another fixed point. As $K_{0}=F\left(a_{0}\right) \leq F\left(a^{\prime}\right)$, we have $K_{1} \leq T\left(F\left(a_{0}\right)\right) \leq T\left(F\left(a^{\prime}\right)\right)=F\left(a^{\prime}\right)$ and by induction, for all $n, K_{n} \leq F\left(a^{\prime}\right)$, hence $F(a)=\sup _{n} K_{n} \leq F\left(a^{\prime}\right)$. This proves $\mathbf{i}$ and ii.

To prove (iii) we need to remark that $\uparrow V\left(F\left(a_{0}\right)\right)$ is directed complete partially ordered soft set with a smallest element $V\left(F\left(a_{0}\right)\right)$ and $S$ maps $\uparrow V\left(F\left(a_{0}\right)\right)$ into itself as $V\left(F\left(a_{0}\right)\right) \leq G(b)$ implies

$$
S(G(b))=S\left(V\left(F\left(a_{0}\right)\right)\right)=V\left(T\left(F\left(a_{0}\right)\right)\right) \geq V\left(F\left(a_{0}\right)\right) .
$$

Hence the restriction of $S$ to $\uparrow V\left(K_{0}\right)$ has a least fixed point and

$$
\begin{aligned}
V(\operatorname{LFP}(T)) & =V\left(\sup _{n} T\left(K_{n}\right)\right), & & \text { by (ii), } \\
& =\sup _{n} V\left(T\left(K_{n}\right)\right), & & \text { as V Soft Scott continuous, } \\
& =\sup _{n} S\left(V\left(F_{n}\right)\right), & & \text { as diagram commutes, } \\
& =\operatorname{LFP}\left(S_{\uparrow} V\left(K_{0}\right)\right), & & \text { by (ii). }
\end{aligned}
$$

Note that, if $V$ is strict, i.e., if $V\left(K_{0}\right)=H_{0}$, then $V(\operatorname{LFP}(T))=\operatorname{LFP}(S)$.

\section{Conclusion}

In this paper, an ordering on soft elements were defined and soft continuity on soft topological spaces were examined. Furthermore, least fixed point theorem for soft Scott continuous functions was proved. From this study, it can be seen that the most of properties of lattice, Scott topology, and Scott continuous function in general can be satisfied by soft lattice, soft Scott topology, and soft Scott continuous function, respectively. But there are some exceptions, for example, classical Scott topology is a $T_{0}$ topological space while soft Scott topology is not a $\mathrm{T}_{0}$ soft topological space. Moreover one can study categorical structures according to this paper on soft sets. 


\section{Acknowledgment}

The authors deeply thank to editor and referees for their valuable comments and special thank to Nazan Çakmak Polat for her helpful corrections that improved this paper. This work is supported by the Scientific Research Project of Muğla Sitkı Koçman University, SRPO (no: 16/073).

\section{References}

[1] M. I. Ali, F. Feng, X.-Y. Liu, W. K. Min, M. Shabir, On some new operations in soft set theory, Comput. Math. Appl., 57 (2009), 1547-1553. 2.6

[2] K. V. Babitha, J. J. Sunil, Soft set relations and functions, Comput. Math. Appl., 60 (2010), 1840-1849. 1, 2.7, $2.8,2.9$

[3] K. V. Babitha, J. J. Sunil, Transitive closures and ordering on soft sets, Comput. Math. Appl., 62 (2011), 2235-2239. 1, $2.10,2.12,2.13,2.14$

[4] N. Çağman, S. Karataş, S. Enginoglu, Soft topology, Comput. Math. Appl., 62 (2011), 351-258. 1, 2.30, 2.31, 2.33

[5] G. Gierz, K. H. Hofmann, K. Keimel, J. D. Lawson, M. Mislove, D. S. Scott, Continuous lattices and domains, Encyclopedia of Mathematics and its Applications, Cambridge University Press, Cambridge, (2003). 1

[6] K. Hrbáček, T. Jech, Introduction to set theory, Second edition, Monographs and Textbooks in Pure and Applied Mathematics, Marcel Dekker, Inc., New York, (1984). 1

[7] P. K. Maji, R. Biswas, A. R. Roy, Soft set theory, Comput. Math. Appl., 45 (2003), 555-562. 1, 2.2, $2.3,2.4$

[8] D. Molodtsov, Soft set theory-first results, Global optimization, control, and games, III, Comput. Math. Appl., 37 (1999), 19-31. 1, 2.1

[9] D.-W. Pei, D.-Q. Miao, From soft sets to information systems, 2005 IEEE International Conference on Granular Computing, 2 (2005), 617-621. 2.5

[10] S. Roy, T. K. Samanta, An introduction of a soft topological spaces Proceeding of UGC sponsored national seminar on recent trends in fuzzy set theory, rough set theory and soft set theory at Uluberia College on 23rd and 24th September, (2011), 9-12. 1, 2.28, 2.29, 2.32

[11] A. F. Sayed, Continuity of partially ordered soft sets via soft Scott topology and soft sobrification, Bull. Math. Sci. Appl., 9 (2014), 79-88. 3, 3.7

[12] B. Tanay, G. Yaylali, New structures on partially ordered soft sets and soft Scott topology, Ann. Fuzzy Math. Inform., 7 (2014), 89-97. 1, 2.11, 2.20, 2.21, 2.22, 2.23, 2.25, 4.1, 4.2, 4.3, 4.5

[13] B. Tanay, G. Yaylalı, Soft Way Below Relation, International Conference on Recent Advanced in Pure and Applied Mathematics, Antalya, Turkey, (2014). 1, 3.1, 3.2, 3.3, 3.4, 3.5, 3.6, 3.7

[14] D. Wardowski, On a soft mapping and its fixed points, Fixed Point Theory Appl., 2013 (2013), 11 pages. 1, 2.16, 2.26, 2.35

[15] G. Yaylalı, B. Tanay, Intervals, Soft Ordered Topology and Some Results, National Mathematics Symposium UMS, (2014). 2.15

[16] G. Yaylalı, B. Tanay, Soft lattices and some results on orderings on soft set, J. Adv. Res. Pure Math., 7 (2015), 45-60. 1, 2.27

[17] G. Yaylalı, B. Tanay, Some new results on orderings on soft sets, J. Inst. Sci. Technol. Dumlupinar Univ., 34 (2015), 5 pages. $1,2.23,2.24$ 